\title{
When to expect the unexpected: uterine ruptures before onset of labour. (Mini-commentary on BJOG-20-0185.R1)
}

\author{
Griet Vandenberghe ${ }^{1}$ \\ ${ }^{1}$ Ghent University Hospital
}

July 1, 2020

Uterine rupture is the feared complication when a uterus scarred by a caesarean section (CS) is labouring. The increased awareness of uterine rupture and its dramatic consequences of perinatal asphyxia has lead to guidelines dictating safe circumstances when undertaking a trial of labour after caesarean section (TOLAC). This complication occurs in an estimated 35 per 10000 women with a previous CS undergoing labour (INOSS study of uterine rupture, BJOG 2019;126:370-381), but is extremely rare before the onset of labour and in the unscarred uterus. Our knowledge of prelabour uterine ruptures is confined merely to a small number of published case reports and case series in the literature.

In this issue of BJOG, Al-Zirqi and colleagues present the results of a retrospective population-based study during 41 years (1967-2008) in Norway describing the characteristics and outcomes of complete uterine rupture before the onset of labour in 8 unscarrred and 22 scarred uteri, besides another 45 partial ruptures in scarred uteri (BJOG $2020 \mathrm{xxxx}$ ). The authors point out that complete ruptures before onset of labour are absolutely rare, but result in a higher number of perinatal deaths. In the unscarred uterus, complete ruptures before onset of labour were related to trauma $(n=3)$, abnormal invasive placenta $(n=2)$ and congenital uterine malformations $(n=2)$ and were unavoidable in most cases. In the scarred uterus, complete ruptures before onset of labour were related to abnormally invasive placenta $(n=4)$, previous complete uterine rupture $(n=3)$ and scars outside the lower uterine segment $(n=17)$. These scars were caused by classical CS $(n=9)$, myomectomy $(n=5)$, tubo-uterine surgery $(n=3)$, uterine septal operation $(n=1)$ and perforation during endometrial ablations $(\mathrm{n}=3)$. Classical $\mathrm{CS}$ by vertical incision has become rare, while laparoscopic and hysteroscopic uterine surgery increase in frequency. A systematic review by Gambacorti-Passerini et al. (Acta Obstetricia et Gynecologica Scandinavica 2016;95:724-734) revealed that the majority of uterine ruptures after prior myomectomy occurred before 36 weeks and before labour $(5 / 330,1.5 \%)$, while only $0.47 \%(2 / 426)$ in women undergoing a trial of labour after myomectomy (TOLAM). The researchers could not reveal any riskfactor (surgical approach, type of myoma, type of hemostasis, suture layers) keeping the risk of uterine rupture following prior myomectomy largely unpredictable. Only case reports and small case series report on uterine ruptures following operative hysteroscopy or tubo-uterine surgery, where perforation is considered a risk factor (Sentilhes et al. Gynecol Obstet Fertil 2006;34(11):1064-70), similar to the 3 cases of endometrial ablation with perforation in the study of Al-Zirqi et al. More large scale studies like this retrospective analysis are needed to understand to what extent uterine surgical procedures contribute to uterine ruptures, during or before onset of labour. In this regard, we expect a report of the International Network of Obstetric Survey Systems (INOSS) who analysed complete uterine ruptures in 10 countries, including 215 uterine ruptures before onset of labour.

This study advocates a higher level of alertness for uterine rupture when taking care for women with congenital uterine abnormalities, with suspected AIP and with prior uterine surgery.

No disclosures: A completed disclosure of interest form is available to view online as supporting informa- 
tion. 